\title{
Error bounds of block sparse signal recovery based on $q$-ratio block constrained minimal singular values
}

\author{
Jianfeng Wang ${ }^{1 *}$, Zhiyong Zhou ${ }^{2}$ and Jun $\mathrm{Yu}^{1}$
}

\begin{abstract}
In this paper, we introduce the $q$-ratio block constrained minimal singular values (BCMSV) as a new measure of measurement matrix in compressive sensing of block sparse/compressive signals and present an algorithm for computing this new measure. Both the mixed $\ell_{2} / \ell_{q}$ and the mixed $\ell_{2} / \ell_{1}$ norms of the reconstruction errors for stable and robust recovery using block basis pursuit (BBP), the block Dantzig selector (BDS), and the group lasso in terms of the $q$-ratio BCMSV are investigated. We establish a sufficient condition based on the $q$-ratio block sparsity for the exact recovery from the noise-free BBP and developed a convex-concave procedure to solve the corresponding non-convex problem in the condition. Furthermore, we prove that for sub-Gaussian random matrices, the q-ratio BCMSV is bounded away from zero with high probability when the number of measurements is reasonably large. Numerical experiments are implemented to illustrate the theoretical results. In addition, we demonstrate that the $q$-ratio BCMSV-based error bounds are tighter than the block-restricted isotropic constant-based bounds.
\end{abstract}

Keywords: Compressive sensing, $q$-ratio block sparsity, $q$-ratio block constrained minimal singular value, Convex-concave procedure

\section{Introduction}

Compressive sensing (CS) $[1,2]$ aims to recover an unknown sparse signal $\mathbf{x} \in \mathbb{R}^{N}$ from $m$ noisy measurements $\mathbf{y} \in \mathbb{R}^{m}$ :

$$
\mathbf{y}=A \mathbf{x}+\boldsymbol{\epsilon}
$$

where $A \in \mathbb{R}^{m \times N}$ is a measurement matrix with $m \ll$ $N$, and $\boldsymbol{\epsilon} \in \mathbb{R}^{m}$ is additive noise such that $\|\boldsymbol{\epsilon}\|_{2} \leq \zeta$ for some $\zeta \geq 0$. It has been proven that if $A$ satisfies the (stable/robust) null space property (NSP) or restricted isometry property (RIP), (stable/robust) recovery can be achieved [3, Chapter 4 and 6]. However, it is computationally hard to verify NSP and compute the restricted isometry constant (RIC) for an arbitrarily chosen $A[4,5]$. To overcome the drawback, a new class of measures for the measurement matrix has been developed during the last decade. To be specific, [6] introduced a new measure called $\ell_{1}$-constrained minimal singular value $(\mathrm{CMSV})$ :

\footnotetext{
*Correspondence: jianfeng.wang@umu.se

'Department of Mathematics and Mathematical Statistics, Umeå University, Umeå, Sweden

Full list of author information is available at the end of the article
}

$\rho_{S}(A)=\min _{\mathbf{z} \neq 0,\|\mathbf{z}\|_{1}^{2} /\|\mathbf{z}\|_{2}^{2} \leq s} \frac{\|A \mathbf{z}\|_{2}}{\|\mathbf{z}\|_{2}}$ and obtained the $\ell_{2}$ recovery error bounds in terms of the proposed measure for the basis pursuit (BP) [7], the Dantzig selector (DS) [8], and the lasso estimator [9]. Afterwards, [10] brought in a variant of the CMSV: $\omega_{\diamond}(A, s)=\min _{\mathbf{z} \neq 0,\|\mathbf{z}\|_{1} /\|\mathbf{z}\|_{\infty} \leq s} \frac{\|A \mathbf{z}\|_{\diamond}}{\|\mathbf{z}\|_{\infty}}$ with $\|\cdot\|_{\diamond}$ denoting a general norm and expressed the $\ell_{\infty}$ recovery error bounds using this quantity. The latest progress concerning the CMSV can be found in $[11,12]$. Zhou and $\mathrm{Yu}$ [11] generalized these two measures to a new measure called $q$-ratio CMSV: $\rho_{q, s}(A)=$ $\min _{\mathbf{z} \neq 0,\left(\|\mathbf{z}\|_{1} /\|\mathbf{z}\|_{q}\right)^{q /(q-1)} \leq s} \frac{\|A \mathbf{z}\|_{2}}{\|\mathbf{z}\|_{q}}$ with $q \in(1, \infty]$ and established both $\ell_{q}$ and $\ell_{1}$ bounds of recovery errors. Zhou and $\mathrm{Yu}$ [12] investigated geometrical property of the $q$-ratio CMSV, which can be used to derive sufficient conditions and error bounds of signal recovery.

In addition to the simple sparsity, a signal $\mathbf{x}$ can also possess a structure called block sparsity where the nonzero elements occur in clusters. It has been shown that using block information in CS can lead to a better signal recovery [13-15]. Analogue to the simple sparsity, 
there are block NSP and block RIP to characterize the measurement matrix in order to guarantee a successful recovery through (1) [16]. Nevertheless, they are still computationally hard to be verified for a given $A$. Thus, it is desirable to develop a computable measure like the CMSV for recovery of simple (non-block) sparse signals. Tang and Nehorai [17] proposed a new measure of the measurement matrix based on the CMSV for block sparse signal recovery and derived the mixed $\ell_{2} / \ell_{\infty}$ and $\ell_{2}$ bounds of recovery errors. In this paper, we extend the $q$-ratio CMSV in [11] to $q$-ratio block CMSV (BCMSV) and generalize the error bounds from the mixed $\ell_{2} / \ell_{\infty}$ and $\ell_{2}$ norms in [17] to mixed $\ell_{2} / \ell_{q}$ with $q \in(1, \infty]$ and mixed $\ell_{2} / \ell_{1}$ norms.

This work includes four main contributions to block sparse signal recovery in compressive sensing: (i) we establish a sufficient condition based on the $q$-ratio block sparsity for the exact recovery from the noise-free block $\mathrm{BP}$ (BBP) and develop a convex-concave procedure to solve the corresponding non-convex problem in the condition; (ii) we introduce the $q$-ratio BCMSV and derive both the mixed $\ell_{2} / \ell_{q}$ and the mixed $\ell_{2} / \ell_{1}$ norms of the reconstruction errors for stable and robust recovery using the BBP, the block DS (BDS), and the group lasso in terms of the $q$-ratio BCMSV; (iii) we prove that for sub-Gaussian random matrices, the $q$-ratio BCMSV is bounded away from zero with high probability when the number of measurements is reasonably large; and (iv) we present an algorithm to compute the $q$-ratio BCMSV for an arbitrary measurement matrix and investigate its properties.

The paper is organized as follows. Section 2 presents our theoretical contributions, including properties of the $q$-ratio block sparsity and the $q$-ratio BCMSV, the mixed $\ell_{2} / \ell_{q}$ norm and the mixed $\ell_{2} / \ell_{1}$ norm reconstruction errors for the BBP, the BDS and the group lasso, and the probabilistic result of the $q$-ratio BCMSV for subGaussian random matrices. Numerical experiments and algorithms are described in Section 3. Section 4 is devoted to conclusion and discussion. All the proofs are left in the Appendix.

\section{Theoretical methodology}

\section{1 q-ratio block sparsity and q-ratio BCMSV - definition} and property

In this section, we introduce the definitions of the $q$-ratio block sparsity and the $q$-ratio BCMSV and present their fundamental properties. A sufficient condition for block sparse signal recovery via the noise-free BBP using the $q$-ratio block sparsity and an inequality for the q-ratio BCMSV are established.

Throughout the paper, we denote vectors by bold lower case letters or bold numbers and matrices by upper case letters. $\mathbf{x}^{T}$ denotes the transpose of a column vector $\mathbf{x}$. For any vector $\mathbf{x} \in \mathbb{R}^{N}$, we partition it into $p$ blocks, each of length $n$, so we have $\mathbf{x}=\left[\mathbf{x}_{1}^{T}, \mathbf{x}_{2}^{T}, \ldots, \mathbf{x}_{p}^{T}\right]^{T}$ and $\mathbf{x}_{i} \in \mathbb{R}^{n}$ denotes the $i$ th block of $\mathbf{x}$. We define the mixed $\ell_{2} / \ell_{0}$ norm $\|\mathbf{x}\|_{2,0}=\sum_{i=1}^{p} 1\left\{\mathbf{x}_{i} \neq \mathbf{0}\right\}$, the mixed $\ell_{2} / \ell_{\infty}$ norm $\|\mathbf{x}\|_{2, \infty}=\max _{1 \leq i \leq p}\left\|\mathbf{x}_{i}\right\|_{2}$, and the mixed $\ell_{2} / \ell_{q}$ norm $\|\mathbf{x}\|_{2, q}=\left(\sum_{i=1}^{p}\left\|\mathbf{x}_{i}\right\|_{2}^{q}\right)^{1 / q}$ for $0<q<\infty$. A signal $\mathbf{x}$ is block $k$-sparse if $\|\mathbf{x}\|_{2,0} \leq k$. [ $p$ ] denotes the set $\{1,2, \cdots, p\}$ and $|S|$ denotes the cardinality of a set $S$. Furthermore, we use $S^{c}$ for the complement $[p] \backslash S$ of a set $S$ in $[p]$. The block support is defined by bsupp $(\mathbf{x}):=\{i \in[p]:$ $\left.\left\|\mathbf{x}_{i}\right\|_{2} \neq 0\right\}$. If $S \subset[p]$, then $\mathbf{x}_{S}$ is the vector coincides with $\mathbf{x}$ on the block indices in $S$ and is extended to zero outside $S$. For any matrix $A \in \mathbb{R}^{m \times N}, \operatorname{ker} A:=\left\{\mathbf{x} \in \mathbb{R}^{N}: A \mathbf{x}=\mathbf{0}\right\}$, $A^{T}$ is the transpose. $\langle\cdot, \cdot\rangle$ is the inner product function.

We first introduce the definition of the $q$-ratio block sparsity and its properties.

Definition 1 ([18]) For any non-zero $\mathbf{x} \in \mathbb{R}^{N}$ and nonnegative $q \notin\{0,1, \infty\}$, the $q$-ratio block sparsity of $\mathbf{x}$ is defined as

$$
k_{q}(\mathbf{x})=\left(\frac{\|\mathbf{x}\|_{2,1}}{\|\mathbf{x}\|_{2, q}}\right)^{\frac{q}{q-1}} .
$$

The cases of $q \in\{0,1, \infty\}$ are evaluated by limits:

$$
\begin{aligned}
& k_{0}(\mathbf{x})=\lim _{q \rightarrow 0} k_{q}(\mathbf{x})=\|\mathbf{x}\|_{2,0} \\
& k_{1}(\mathbf{x})=\lim _{q \rightarrow 1} k_{q}(\mathbf{x})=\exp \left(H_{1}(\pi(\mathbf{x}))\right) \\
& k_{\infty}(\mathbf{x})=\lim _{q \rightarrow \infty} k_{q}(\mathbf{x})=\frac{\|\mathbf{x}\|_{2,1}}{\|\mathbf{x}\|_{2, \infty}} .
\end{aligned}
$$

Here, $\pi(\mathbf{x}) \in \mathbb{R}^{p}$ with entries $\pi_{i}(\mathbf{x})=\left\|\mathbf{x}_{i}\right\|_{2} /\|\mathbf{x}\|_{2,1}$ and $H_{1}$ is the ordinary Shannon entropy $H_{1}(\pi(\mathbf{x}))=$ $-\sum_{i=1}^{p} \pi_{i}(\mathbf{x}) \log \pi_{i}(\mathbf{x})$.

This is an extension of the sparsity measures proposed in $[19,20]$, where estimation and statistical inference via $\alpha$-stable random projection method were investigated. In fact, this kind of sparsity measure is based on entropy, which measures energy of blocks of $\mathbf{x}$ via $\pi_{i}(\mathbf{x})$. Formally, we can express the $q$-ratio block sparsity by

$$
k_{q}(\mathbf{x})= \begin{cases}\exp \left(H_{q}(\pi(\mathbf{x}))\right) & \text { if } \mathbf{x} \neq \mathbf{0} \\ 0 & \text { if } \mathbf{x}=\mathbf{0},\end{cases}
$$

where $H_{q}$ is the Rényi entropy of order $q \in[0, \infty][21$, 22]. When $q \notin\{0,1, \infty\}$, the Rényi entropy is given by $H_{q}(\pi(\mathbf{x}))=\frac{1}{1-q} \log \left(\sum_{i=1}^{p} \pi_{i}(\mathbf{x})^{q}\right)$, and for the cases of $q \in\{0,1, \infty\}$, the Rényi entropy is evaluated by limits and results in (3), (4), and (5), respectively.

Next, we present a sufficient condition for the exact recovery via the noise-free BBP in terms of the $q$-ratio block sparsity. Recall that when the true signal $\mathbf{x}$ is block $k$ sparse, the sufficient and necessary condition for the exact recovery via the noise-free BBP: 


$$
\min _{\mathbf{z} \in \mathbb{R}^{N}}\|\mathbf{z}\|_{2,1} \text { s.t. } A \mathbf{z}=A \mathbf{x}
$$

in terms of the block NSP of order $k$ was given by $[16,23]$

$$
\left\|\mathbf{z}_{S}\right\|_{2,1}<\left\|\mathbf{z}_{S^{c}}\right\|_{2,1}, \forall \mathbf{z} \in \operatorname{ker} A \backslash\{\mathbf{0}\}, S \subset[p] \text { and }|S| \leq k .
$$

Proposition 1 If $\mathbf{x}$ is block $k$-sparse and there exists at least one $q \in(1, \infty]$ such that $k$ is strictly less than

$$
\min _{\mathbf{z} \in \operatorname{ker} A \backslash\{\mathbf{0}\}} 2^{\frac{q}{1-q}} k_{q}(\mathbf{z}),
$$

then the unique solution to problem (7) is the true signal $\mathbf{x}$.

Remark 1 The proof can be found in A.1 in Appendix. This proposition is an extension of Proposition 1 in [11] from simple sparse signals to block sparse signals. In Section 3.1, we adopt a convex-concave procedure algorithm to solve (8) approximately.

Now, we are ready to present the definition of the $q$-ratio BCMSV, which is developed based on the $q$-ratio block sparsity.

Definition 2 For any real number $s \in[1, p], q \in(1, \infty]$ and matrix $A \in \mathbb{R}^{m \times N}$, the $q$-ratio block constrained minimal singular value (BCMSV) of $A$ is defined as

$$
\beta_{q, s}(A)=\min _{\mathbf{z} \neq \mathbf{0}, k_{q}(\mathbf{z}) \leq s} \frac{\|A \mathbf{z}\|_{2}}{\|\mathbf{z}\|_{2, q}} .
$$

Remark 2 For measurement matrix $A$ with unit norm columns, it is obvious that $\beta_{q, s}(A) \leq 1$ since $\left\|A \mathbf{e}_{i}\right\|_{2}=1$, $\left\|\mathbf{e}_{i}\right\|_{2, q}=1$, and $k_{q}\left(\mathbf{e}_{i}\right)=1$, where $\mathbf{e}_{i}$ is the ith canonical basis for $\mathbb{R}^{N}$. Moreover, when $q$ and $A$ are fixed, $\beta_{q, s}(A)$ is non-increasing with respect to $s$. Besides, it is worth noticing that the q-ratio BCMSV depends also on the block size n, we choose to not show this parameter for the sake of simplicity. Another interesting finding is that for any $\alpha \in \mathbb{R}$, we have $\beta_{q, s}(\alpha A)=|\alpha| \beta_{q, s}(A)$. This fact together with Theorem 1 in Section 2.2 implies that in the case of adopting a measurement matrix $\alpha$ A, increasing the measurement energy through $|\alpha|$ will proportionally reduce the mixed $\ell_{2} / \ell_{q}$ norm of reconstruction errors. Comparing to the block RIP [16], there are three main advantages by using the q-ratio BCMSV:

- It is computable (see the algorithm in Section 3.2).

- The proof procedures and results of recovery error bounds are more concise (details in Section 2.2).

- The q-ratio BCMSV-based recovery bounds are smaller (better) than the block RIC-based bounds as shown in Section 3.3 (see also [11, 17], for another two specific examples).

As for different $q$, we have the following important inequality, which plays a crucial role in deriving the probabilistic behavior of $\beta_{q, s}(A)$ via the existing results established in [17].

Proposition 2 If $1<q_{2} \leq q_{1} \leq \infty$, then for any real number $1 \leq s \leq p^{1 / \tilde{q}}$ with $\tilde{q}=\frac{q_{2}\left(q_{1}-1\right)}{q_{1}\left(q_{2}-1\right)}$, we have

$$
\beta_{q_{1}, s}(A) \geq \beta_{q_{2}, s^{\tilde{q}}}(A) \geq s^{-\tilde{q}} \beta_{q_{1}, s \tilde{q}}(A) .
$$

Remark 3 The proof can be found in A.2 in Appendix. Let $q_{1}=\infty$ and $q_{2}=2$ (thus, $\tilde{q}=2$ ), we have $\beta_{\infty, s}(A) \geq$ $\beta_{2, s^{2}}(A) \geq \frac{1}{s^{2}} \beta_{\infty, s^{2}}(A)$. If $q_{1} \geq q_{2}>1$, then $\tilde{q}=\frac{q_{2}\left(q_{1}-1\right)}{q_{1}\left(q_{2}-1\right)}=$ $1+\frac{q_{1}-q_{2}}{q_{1}\left(q_{2}-1\right)} \geq 1$, so $\beta_{q_{2}, s \tilde{q}}(A) \leq \beta_{q_{2}, s}(A)$. Similarly, we have for any $t \in[1, p] \beta_{q_{2}, t}(A) \geq \frac{1}{t} \beta_{q_{1}, t}(A)$ by letting $t=s^{\tilde{q}}$ in (10). Based on these facts, we can not obtain the monotonicity with respect to $q$ when $s$ and $A$ are fixed. However, since for any $\mathbf{z} \in \mathbb{R}^{N}$ with $p$ blocks, $k_{q}(\mathbf{z}) \leq p$, it holds trivially that $\beta_{q, p}(A)$ is non-decreasing with respect to $q$ by using the non-increasing property of the mixed $\ell_{2} / \ell_{q}$ norm.

\subsection{Recovery error bounds}

In this section, we derive the recovery error bounds in terms of the mixed $\ell_{2} / \ell_{q}$ norm and the mixed $\ell_{2} / \ell_{1}$ norm via the $q$-ratio BCMSV of the measurement matrix. We focus on three renowned convex relaxation algorithms for block sparse signal recovery from (1): the BBP, the BDS, and the group lasso.

BBP: $\min _{\mathbf{z} \in \mathbb{R}^{N}}\|\mathbf{z}\|_{2,1}$ s.t. $\|\mathbf{y}-A \mathbf{z}\|_{2} \leq \zeta$.

BDS: $\min _{\mathbf{z} \in \mathbb{R}^{N}}\|\mathbf{z}\|_{2,1}$ s.t. $\left\|A^{T}(\mathbf{y}-A \mathbf{z})\right\|_{2, \infty} \leq \mu$.

Group lasso: $\min _{\mathbf{z} \in \mathbb{R}^{N}} \frac{1}{2}\|\mathbf{y}-A \mathbf{z}\|_{2}^{2}+\mu\|\mathbf{z}\|_{2,1}$.

Here, $\zeta$ and $\mu$ are parameters used in the constraints to control the noise level. We first present the following main results of recovery error bounds for the case when the true signal $\mathbf{x}$ is block $k$-sparse.

Theorem 1 Suppose $\mathbf{x}$ is block k-sparse. For any $q \in$ $(1, \infty]$, we have

1) If $\|\boldsymbol{\epsilon}\|_{2} \leq \zeta$, then the solution $\hat{\mathbf{x}}$ to the BBP obeys

$$
\begin{aligned}
\|\hat{\mathbf{x}}-\mathbf{x}\|_{2, q} & \leq \frac{2 \zeta}{\beta_{q, 2^{\frac{q}{q-1}} k}(A)}, \\
\|\hat{\mathbf{x}}-\mathbf{x}\|_{2,1} \leq & \frac{4 k^{1-1 / q_{\zeta}}}{\beta_{q, 2^{\frac{q}{q-1}} k}(A)} .
\end{aligned}
$$


2) If the noise $\boldsymbol{\epsilon}$ in the BDS satisfies $\left\|A^{T} \boldsymbol{\epsilon}\right\|_{2, \infty} \leq \mu$, then the solution $\hat{\mathbf{x}}$ to the BDS obeys

$$
\begin{aligned}
\|\hat{\mathbf{x}}-\mathbf{x}\|_{2, q} & \leq \frac{4 k^{1-1 / q}}{\beta^{2}{ }_{q, 2^{\frac{q}{q-1}} k}(A)} \mu, \\
\|\hat{\mathbf{x}}-\mathbf{x}\|_{2,1} & \leq \frac{8 k^{2-2 / q}}{\beta^{2}{ }_{q, 2^{\frac{q}{q-1}} k}(A)} \mu .
\end{aligned}
$$

3) If the noise $\boldsymbol{\epsilon}$ in the group lasso satisfies $\left\|A^{T} \boldsymbol{\epsilon}\right\|_{2, \infty} \leq \kappa \mu$ for some $\kappa \in(0,1)$, then the solution $\hat{\mathbf{x}}$ to the group lasso obeys

$$
\begin{aligned}
& \|\hat{\mathbf{x}}-\mathbf{x}\|_{2, q} \leq \frac{1+\kappa}{1-\kappa} \cdot \frac{2 k^{1-1 / q}}{\beta^{2}{ }_{q,\left(\frac{2}{1-\kappa}\right)^{\frac{q}{q-1}} k}(A)} \mu, \\
& \|\hat{\mathbf{x}}-\mathbf{x}\|_{2,1} \leq \frac{1+\kappa}{(1-\kappa)^{2}} \cdot \frac{4 k^{2-2 / q}}{\beta^{2}\left(\frac{2}{1-\kappa}\right)^{\frac{q}{q-1} k}(A)} \mu .
\end{aligned}
$$

Remark 4 The proof can be found in A.3 in Appendix. Obviously, if $\beta_{q, 2^{\frac{q}{q-1}} k}(A) \neq 0$ in (11) and (12), then the noise free BBP (7) can uniquely recover any block $k$-sparse signal by letting $\zeta=0$.

Remark 5 The mixed $\ell_{2} / \ell_{q}$ norm error bounds are generalized from the existing results in [17] $(q=2$ and $\infty)$ to any $1<q \leq \infty$ and from [11] (simple sparse signal recovery) to block sparse signal recovery. The mixed $\ell_{2} / \ell_{q}$ norm error bounds depend on the q-ratio BCMSV of the measurement matrix $A$, which is bounded away from zero for sub-Gaussian random matrix and can be computed approximately by using a specific algorithm, which are discussed later.

Remark 6 As shown in literature, the block RIC-based recovery error bounds for the $B B P$ [16], the BDS [24], and the group lasso [25] are complicated. In contrast, as presented in this theorem, the q-ratio BCMSV-based bounds are much more concise and corresponding derivations are much less complicated, which are given in the Appendix.

Next, we extend Theorem 1 to the case when the signal is block compressible, in the sense that it can be approximated by a block $k$-sparse signal. Given a block compressible signal $\mathbf{x}$, let the mixed $\ell_{2} / \ell_{1}$ error of the best block $k$-sparse approximation of $\mathbf{x}$ be $\phi_{k}(\mathbf{x})=\inf _{\mathbf{z} \in \mathbb{R}^{N},\|\mathbf{z}\|_{2,0}=k} \| \mathbf{x}-$ $\mathbf{z} \|_{2,1}$, which measures how close $\mathbf{x}$ is to the block $k$-sparse signal.

Theorem 2 Suppose that $\mathbf{x}$ is block compressible. For any $1<q \leq \infty$, we have
1) If $\|\boldsymbol{\epsilon}\|_{2} \leq \zeta$, then the solution $\hat{\mathbf{x}}$ to the BBP obeys

$$
\begin{aligned}
\|\hat{\mathbf{x}}-\mathbf{x}\|_{2, q} & \leq \frac{2 \zeta}{\beta \frac{q}{q, 4^{\frac{q}{q-1} k}}(A)}+k^{1 / q-1} \phi_{k}(\mathbf{x}), \\
\|\hat{\mathbf{x}}-\mathbf{x}\|_{2,1} & \leq \frac{4 k^{1-1 / q_{\zeta}}}{\beta_{q, 4^{\frac{q}{q-1}} k}(A)}+4 \phi_{k}(\mathbf{x}) .
\end{aligned}
$$

2) If the noise $\boldsymbol{\epsilon}$ in the BDS satisfies $\left\|A^{T} \boldsymbol{\epsilon}\right\|_{2, \infty} \leq \mu$, then the solution $\hat{\mathbf{x}}$ to the BDS obeys

$$
\begin{aligned}
\|\hat{\mathbf{x}}-\mathbf{x}\|_{2, q} & \leq \frac{8 k^{1-1 / q}}{\beta^{2} \frac{q}{q, 4^{\frac{q}{q-1}} k}(A)} \mu+k^{1 / q-1} \phi_{k}(\mathbf{x}), \\
\|\hat{\mathbf{x}}-\mathbf{x}\|_{2,1} & \leq \frac{16 k^{2-2 / q}}{\beta^{2} \operatorname{q,4}^{\frac{q}{q-1} k}(A)} \mu+4 \phi_{k}(\mathbf{x}) .
\end{aligned}
$$

3) If the noise $\boldsymbol{\epsilon}$ in the group lasso satisfies $\left\|A^{T} \boldsymbol{\epsilon}\right\|_{2, \infty} \leq \kappa \mu$ for some $\kappa \in(0,1)$, then the solution $\hat{\mathbf{x}}$ to the group lasso obeys

$$
\begin{aligned}
\|\hat{\mathbf{x}}-\mathbf{x}\|_{2, q} & \leq \frac{1+\kappa}{1-\kappa} \cdot \frac{4 k^{1-1 / q}}{\beta^{2}} \mu+k^{1 / q-1} \phi_{k}(\mathbf{x}), \\
\|\hat{\mathbf{x}}-\mathbf{x}\|_{2,1} & \leq \frac{1+\kappa}{(1-\kappa)^{\frac{q}{q-1}} k} \cdot \frac{8 k^{2-2 / q}}{\beta^{2}\left(\frac{4}{1-\kappa}\right)^{\frac{q}{q-1} k}(A)} \mu+\frac{4}{1-\kappa} \phi_{k}(\mathbf{x}) .
\end{aligned}
$$

Remark 7 The proof can be found in A.4 in Appendix. All the error bounds consist of two components, one is caused by the measurement error, and another one is due to the sparsity defect.

Remark 8 Comparing to Theorem 1, we need stronger conditions to achieve the valid error bounds. Concisely, we require $\beta_{q, 4^{\frac{q}{q-1}} k}(A)>0, \beta_{q, 4^{\frac{q}{q-1}} k}(A)>0$ and $\beta_{q,\left(\frac{4}{1-\kappa}\right)^{\frac{q}{q-1}} k}(A)>0$ for the BBP, BDS, and group lasso in the block compressible case, while $\beta_{q, 2^{\frac{q}{q-1} k}}(A)>0$, $\beta_{q, 2^{\frac{q}{q-1} k}}(A)>0$ and $\beta_{q,\left(\frac{2}{1-\kappa}\right)^{\frac{q}{q-1} k}}(A)>0$ in the block sparse case, respectively.

\subsection{Random matrices}

In this section, we study the properties of the $q$-ratio BCMSV of sub-Gaussian random matrix. A random vector $\mathbf{x} \in \mathbb{R}^{N}$ is called isotropic and sub-Gaussian with constant $L$ if it holds for all $\mathbf{u} \in \mathbb{R}^{N}$ that $E|\langle\mathbf{x}, \mathbf{u}\rangle|^{2}=\|\mathbf{u}\|_{2}^{2}$ and $P(|\langle\mathbf{x}, \mathbf{u}\rangle| \geq t) \leq 2 \exp \left(-\frac{t^{2}}{L\|\mathbf{u}\|_{2}}\right)$. Then, as shown in Theorem 2 of [17], we have the following lemma. 
Lemma 1 ([17]) Suppose the rows of the scaled measurement matrix $\sqrt{m} A$ to be i.i.d isotropic and sub-Gaussian random vectors with constant $L$. Then, there exists constants $c_{1}$ and $c_{2}$ such that for any $\eta>0$ and $m \geq 1$ satisfying

$$
m \geq c_{1} \frac{L^{2} s(n+\log p)}{\eta^{2}},
$$

we have

$$
\mathbb{E}\left|1-\beta_{2, s}(A)\right| \leq \eta
$$

and

$$
\mathbb{P}\left(\beta_{2, s}(A) \geq 1-\eta\right) \geq 1-\exp \left(-c_{2} \eta^{2} \frac{m}{L^{4}}\right) .
$$

Then, as a direct consequence of Proposition 2 (i.e., if $1<q<2, \beta_{q, s}(A) \geq s^{-1} \beta_{2, s}(A)$; if $2 \leq q \leq$ $\infty, \beta_{q, s}(A) \geq \beta_{2, \frac{2(q-1)}{q}}(A)$.) and Lemma 1, we have the following probabilistic statements for $\beta_{q, s}(A)$.

Theorem 3 Under the assumptions and notations of Lemma 1, it holds that

1) When $1<q<2$, there exist constants $c_{1}$ and $c_{2}$ such that for any $\eta>0$ and $m \geq 1$ satisfying

$$
m \geq c_{1} \frac{L^{2} s(n+\log p)}{\eta^{2}},
$$

we have

$$
\begin{aligned}
\mathbb{E}\left[\beta_{q, s}(A)\right] & \geq s^{-1}(1-\eta), \\
\mathbb{P}\left(\beta_{q, s}(A)\right. & \left.\geq s^{-1}(1-\eta)\right) \geq 1-\exp \left(-c_{2} \eta^{2} \frac{m}{L^{4}}\right) .
\end{aligned}
$$

2) When $2 \leq q \leq \infty$, there exist constants $c_{1}$ and $c_{2}$ such that for any $\eta>0$ and $m \geq 1$ satisfying

$$
m \geq c_{1} \frac{L^{2} s^{\frac{2(q-1)}{q}}(n+\log p)}{\eta^{2}},
$$

we have

$$
\begin{aligned}
\mathbb{E}\left[\beta_{q, s}(A)\right] & \geq 1-\eta, \\
\mathbb{P}\left(\beta_{q, s}(A)\right. & \geq 1-\eta) \geq 1-\exp \left(-c_{2} \eta^{2} \frac{m}{L^{4}}\right) .
\end{aligned}
$$

Remark 9 Theorem 3 shows that for sub-Gaussian random matrix, the q-ratio BCMSV is bounded away from zero as long as the number of measurements is large enough. Sub-Gaussian random matrices include Gaussian and Bernoulli ensembles.

\section{Numerical experiments and results}

In this section, we introduce a convex-concave method to solve the sufficient condition (8) so as to achieve the maximal block sparsity $k$ and present an algorithm to compute the $q$-ratio BCMSV. We also conduct comparisons between the $q$-ratio BCMSV-based bounds and block RIC-based bounds through the BBP.

\subsection{Solving the optimization problem (8)}

According to Proposition 1, given a $q \in(1, \infty]$, we need to solve the optimization problem (8) to obtain the maximal block sparsity $k$ which guaranties that all block $k$-sparse signals can be uniquely recovered by (7). Solving (8) is equivalent to solve the problem:

$$
\max _{\mathbf{z} \in \mathbb{R}^{N}}\|\mathbf{z}\|_{2, q} \text { s.t. } A \mathbf{z}=0 \text { and }\|\mathbf{z}\|_{2,1} \leq 1 .
$$

However, maximizing mixed $\ell_{2} / \ell_{q}$ norm over a polyhedron is non-convex. Here, we adopt the convex-concave procedure (CCP) (see [26] for details) to solve the problem (27) for any $q \in(1, \infty]$. The algorithm is presented as follows:

$$
\begin{aligned}
& \text { Algorithm: CCP to solve (27). } \\
& \text { Give an initial point to } \mathbf{z}_{l} \text { with } l=0 \text {. } \\
& \text { Iterate } \\
& \text { 1. Linearity. Approximate }\|\mathbf{z}\|_{2, q} \text { using the first order } \\
& \text { Taylor expansion } \\
& \|\mathbf{z}\|_{2, q}=\left\|\mathbf{z}_{l}\right\|_{2, q}+\nabla\left(\|\mathbf{z}\|_{2, q}\right)_{\mathbf{z}=\mathbf{z}_{l}}^{T}\left(\mathbf{z}-\mathbf{z}_{l}\right) \\
& =\left\|\mathbf{z}_{l}\right\|_{2, q}+\left[\left\|\mathbf{z}_{l}\right\|_{2, q}^{1-q}\left\|\mathbf{z}_{l_{b}}\right\|_{2}^{q-2} \mathbf{z}_{l}\right]^{T}\left(\mathbf{z}-\mathbf{z}_{l}\right), \\
& \text { where } \mathbf{z}_{l_{b}}=[\underbrace{\left\|\mathbf{z}_{l_{1}}\right\|_{2}, \cdots,\left\|\mathbf{z}_{l_{1}}\right\|_{2}}_{n}, \underbrace{\left\|\mathbf{z}_{l_{2}}\right\|_{2}, \cdots,\left\|\mathbf{z}_{l_{2}}\right\|_{2}}_{n}, \\
& \cdots, \underbrace{\left.\left\|\mathbf{z}_{l_{p}}\right\|_{2}, \cdots,\left\|\mathbf{z}_{l_{p}}\right\|_{2}^{n}\right]}_{n} \text { with }\left\|\mathbf{z}_{l_{i}}\right\|_{2} \text { denoting the } \ell_{2} \\
& \max _{\mathbf{z} \in \mathbb{R}^{N}}\left\|\mathbf{z}_{l}\right\|_{2, q}+\left[\left\|\mathbf{z}_{l}\right\|_{2, q}^{1-q}\left\|\mathbf{z}_{l_{b}}\right\|_{2}^{q-2} \mathbf{z}_{l}\right]^{T}\left(\mathbf{z}-\mathbf{z}_{l}\right) \\
& \text { s.t. } A \mathbf{z}=0,\|\mathbf{z}\|_{2,1} \leq 1 \text {. }
\end{aligned}
$$

We implement the algorithm to solve (27) under the following settings. Let $A$ be either Bernoulli or Gaussian random matrix with $N=256$, varying $m$, block size $n$, and q. Specifically, $m=64,128,192, n=1,2,4,8$, and $q=$ $2,4,16,128$, respectively. The results are summarized in Table 1 . Note that when $n=1$, the algorithm (28) is identical to the one in [11]. The main findings are as follows: (i) by comparing the results between Bernoulli and Gaussian random matrices under the same settings, there is no substantial difference. Thus, we can now merely focus on the left part of the table, i.e., Bernoulli random matrix part; (ii) it can be seen that the results are not monotone with 
Table 1 Maximal sparsity levels from the CCP algorithm for both Bernoulli and Gaussian random matrices with $N=256$ and different combinations of $n, m$, and $q$

\begin{tabular}{|c|c|c|c|c|c|c|c|c|c|}
\hline \multirow{2}{*}{$n$} & \multirow{2}{*}{$m$} & \multicolumn{4}{|c|}{ Bernoulli random matrix } & \multicolumn{4}{|c|}{ Gaussian random matrix } \\
\hline & & $q=2$ & $q=4$ & $q=16$ & $q=128$ & $q=2$ & $q=4$ & $q=16$ & $q=128$ \\
\hline \multirow[t]{3}{*}{1} & 64 & 4 & 4 & 3 & 2 & 4 & 4 & 3 & 3 \\
\hline & 128 & 12 & 9 & 6 & 5 & 13 & 12 & 7 & 6 \\
\hline & 192 & 23 & 22 & 12 & 10 & 23 & 20 & 11 & 10 \\
\hline \multirow[t]{3}{*}{2} & 64 & 3 & 3 & 2 & 2 & 3 & 3 & 2 & 2 \\
\hline & 128 & 9 & 7 & 5 & 4 & 9 & 7 & 5 & 4 \\
\hline & 192 & 16 & 16 & 10 & 8 & 14 & 14 & 9 & 8 \\
\hline \multirow[t]{3}{*}{4} & 64 & 2 & 2 & 1 & 1 & 2 & 2 & 2 & 2 \\
\hline & 128 & 5 & 5 & 4 & 3 & 5 & 5 & 3 & 3 \\
\hline & 192 & 9 & 10 & 6 & 5 & 9 & 10 & 7 & 6 \\
\hline \multirow[t]{3}{*}{8} & 64 & 1 & 1 & 1 & 1 & 1 & 1 & 1 & 1 \\
\hline & 128 & 3 & 3 & 2 & 2 & 2 & 3 & 3 & 2 \\
\hline & 192 & 5 & 6 & 4 & 4 & 5 & 6 & 4 & 4 \\
\hline
\end{tabular}

respect to $q$ (see the row with $n=4, m=192$ ), which verifies the conclusion in Remark 3; (iii) when $m$ is the only variable, it is easy to notice that the maximal block sparsity increases as $m$ increases; and (iv) conversely, when $n$ is the only variable, the maximal block sparsity decreases as $n$ increases, which is in line with the main result in ([27], Theorem 3.1).

\subsection{Computing the $q$-ratio BCMSVs}

Computing the $q$-ratio BCMSV (9) is equivalent to solve

$$
\min _{\mathbf{z} \in \mathbb{R}^{N}}\|A \mathbf{z}\|_{2} \text { s.t. }\|\mathbf{z}\|_{2,1} \leq s^{\frac{q-1}{q}},\|\mathbf{z}\|_{2, q}=1 .
$$

Since the constraint set is not convex, this is a nonconvex optimization problem. In order to solve (29), we use Matlab function fmincon as in [11] and define $\mathbf{z}=$ $\mathbf{z}^{+}-\mathbf{z}^{-}$with $\mathbf{z}^{+}=\max (\mathbf{z}, 0)$ and $\mathbf{z}^{-}=\max (-\mathbf{z}, 0)$. Consequently, (29) can be reformulated to:

$$
\begin{aligned}
& \min _{\mathbf{z}^{+}, \mathbf{z}^{-} \in \mathbb{R}^{N}}\left(\mathbf{z}^{+}-\mathbf{z}^{-}\right)^{T} A^{T} A\left(\mathbf{z}^{+}-\mathbf{z}^{-}\right) \\
& \text {s.t. }\left\|\mathbf{z}^{+}-\mathbf{z}^{-}\right\|_{2,1}-s^{\frac{q-1}{q}} \leq 0, \\
&\left\|\mathbf{z}^{+}-\mathbf{z}^{-}\right\|_{2, q}=1, \\
& \mathbf{z}^{+} \geq 0, \mathbf{z}^{-} \geq 0 .
\end{aligned}
$$

Due to the existence of local minima, we perform an experiment to decide a reasonable number of iterations needed to achieve the "global" minima shown in Fig. 1. In the experiment, we calculate the $q$-ratio BCMSV of a fixed unit norm columns Bernoulli random matrix of size $40 \times 64, n=s=4$, and varying $q=2,4,8$, respectively. Fifty iterations are carried out for each $q$. The figure shows that after about 30 experiments, the estimate of $\beta_{q, s}$, $\hat{\beta}_{q, s}$, becomes convergent, so in the following experiments, we repeat the algorithm 40 times and choose the smallest value $\hat{\beta}_{q, s}$ as the "global" minima. We test indeed to vary $m, s, n$, respectively, all indicate 40 is a reasonable number to be chosen (not shown).

Next, we illustrate the properties of $\beta_{q, s}$, which have been pointed out in Remarks 2 and 3, through experiments. We set $N=64$ with three different block sizes $n=1,4,8$ (i.e., number of blocks $p=64,16,8$ ), three different $m=40,50,60$, three different $q=2,4,8$, and three different $s=2,4,8$. Unit norm columns Bernoulli random matrices are used. Results are listed in Table 2. They are inline with the theoretical results:

(i) $\beta_{q, s}$ increases as $m$ increases for all cases given that other parameters are fixed.

(ii) $\beta_{q, s}$ decreases as $s$ increases for most of cases given that other parameters are fixed. There are exceptions when $m=40, n=8$ with $s=4$, and $s=8$ under $q=4,8$, respectively. However, the difference is about 0.0002 , which is possibly caused by numerical approximation.

(iii) Monotonicity of $\beta_{q, s}$ does not hold with respect to $q$ even given that other parameters are fixed.

\subsection{Comparing error bounds}

Here, we compare the $q$-ratio BCMSV-based bounds against the block RIC-based bounds from the BBP under different settings. The block RIC-based bound is

$$
\|\hat{x}-x\|_{2} \leq \frac{4 \sqrt{1+\delta_{2 k}(A)}}{1-(1+\sqrt{2}) \delta_{2 k}(A)} \zeta,
$$

if $A$ satisfies the block RIP of order $2 k$, i.e., the block RIC $\delta_{2 k}(A)<\sqrt{2}-1[14,17]$. By using the Hölder's inequality, one can obtain the mixed $\ell_{2} / \ell_{q}$ norm

$$
\|\hat{x}-x\|_{2, q} \leq \frac{4 \sqrt{1+\delta_{2 k}(A)}}{1-(1+\sqrt{2}) \delta_{2 k}(A)} k^{1 / q-1 / 2} \zeta,
$$

for $0<q \leq 2$.

We compare the two bounds (32) and (12). Without loss of generality, let $\zeta=1 . \delta_{2 k}(A)$ is approximated using Monte Carlo simulations. Specifically, we randomly choose 1000 sub-matrices of $A \in \mathbb{R}^{m \times N}$ of size $m \times 2 n k$ to compute $\delta_{2 k}(A)$ using the maximum of $\max \left(\sigma_{\max }^{2}-1,1-\sigma_{\min }^{2}\right)$ among all sampled submatrices. It turns out that this approximated block RIC is always smaller than or equal to the exact block RIC; thus, the error bounds based on the exact block RIC are always larger than those based on the approximated block RIC. Therefore, it would be enough to show that the $q$-ratio BCMSV gives a sharper error bound than the approximated block RIC.

We use unit norm columns sub-matrices of a rowrandomly-permuted Hadamard matrix (an orthogonal 


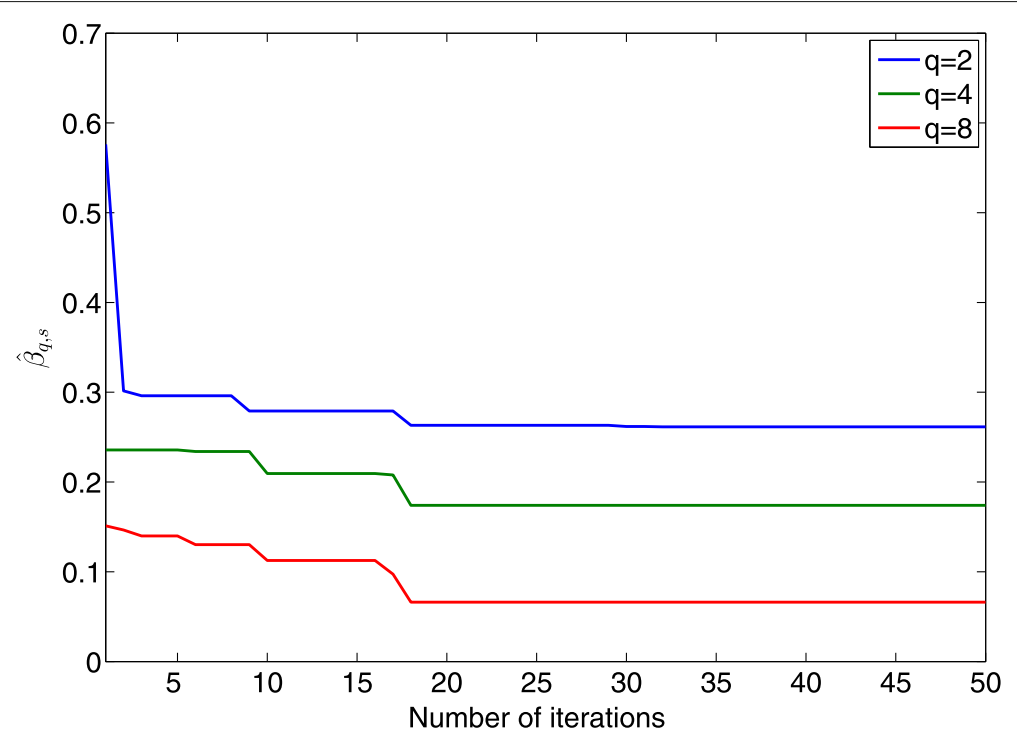

Fig. 1 -ratio BCMSVs calculated for a Bernoulli random matrix of size $40 \times 64$ with $n=4, s=4$, and $q=2,4,8$ as a function of number of experiments

Bernoulli matrix) with $N=64, k=1,2,4, n=1,2$, $q=1.8$, and a variety of $m \leq 64$ to approximate the $q$ ratio BCMSV and the block RIC. Besides the Hadamard matrix, we also test Bernoulli random matrices and Gaussian random matrices with different configurations, which only return very fewer qualified block RICs. In the simulation results of [17], the authors showed that under all considered cases for Gaussian random matrices, $\delta_{2 k}(A)>$ $\sqrt{2}-1$, which is coincident with our finding. Figure 2 shows that the $q$-ratio BCMSV-based bounds are smaller than those based on the approximated block RIC. Note that when $m$ approaches $N, \beta_{q, s}(A) \rightarrow 1$ and $\delta_{2 k}(A) \rightarrow 0$, as a result, the $q$-ratio BCMSV-based bounds are smaller than 2.2, while the block RIC-based bounds are larger than or equal to 4 .

\section{Conclusion and discussion}

In this study, we introduced the $q$-ratio block sparsity measure and the $q$-ratio BCMSV. Theoretically, through the $q$-ratio block sparsity measure and the $q$-ratio BCMSV, we (i) established the sufficient condition for the unique noise-free BBP recovery; (ii) derived both the mixed $\ell_{2} / \ell_{q}$ norm and the mixed $\ell_{2} / \ell_{1}$ norm bounds of recovery errors for the BBP, the BDS, and the group lasso estimator; and (iii) proved the $q$-ratio BCMSV is bounded away from zero if the number of measurements is relatively large for sub-Gaussian random matrix. Afterwards, we used numerical experiments via two algorithms to illustrate theoretical results. In addition, we demonstrated that the $q$-ratio BCMSV-based error bounds are much tighter than those based on block RIP through simulations.

Table 2 The $q$-ratio BCMSVs with varying $m, n, p, q$, and $s$

\begin{tabular}{|c|c|c|c|c|c|c|c|c|c|c|c|}
\hline \multirow{2}{*}{$m$} & \multirow{2}{*}{$n$} & \multirow{2}{*}{$p$} & \multicolumn{3}{|l|}{$q=2$} & \multicolumn{3}{|l|}{$q=4$} & \multicolumn{3}{|l|}{$q=8$} \\
\hline & & & $s=2$ & $s=4$ & $s=8$ & $s=2$ & $s=4$ & $s=8$ & $s=2$ & $s=4$ & $s=8$ \\
\hline \multirow[t]{3}{*}{40} & 1 & 64 & 0.7025 & 0.5058 & 0.2732 & 0.7579 & 0.5495 & 0.1863 & 0.7223 & 0.3954 & 0.0726 \\
\hline & 4 & 16 & 0.4953 & 0.2614 & $3.5 e-04$ & 0.5084 & 0.1741 & $5.1 e-04$ & 0.4592 & 0.0662 & $5.2 e-04$ \\
\hline & 8 & 8 & 0.3240 & 0.0256 & $5.1 e-04$ & 0.2987 & $4.1 e-04$ & $6.1 e-04$ & 0.2492 & $3.9 e-04$ & $6.6 e-04$ \\
\hline \multirow[t]{3}{*}{50} & 1 & 64 & 0.7199 & 0.5169 & 0.3547 & 0.7753 & 0.5766 & 0.2676 & 0.7366 & 0.5250 & 0.1573 \\
\hline & 4 & 16 & 0.5389 & 0.3137 & 0.0767 & 0.5235 & 0.2975 & 0.0015 & 0.4870 & 0.1816 & $9.5 e-04$ \\
\hline & 8 & 8 & 0.4324 & 0.1274 & $9.9 e-04$ & 0.3783 & 0.0398 & 0.0010 & 0.3190 & $8.5 e-04$ & $9.3 e-04$ \\
\hline \multirow[t]{3}{*}{60} & 1 & 64 & 0.7345 & 0.5835 & 0.4316 & 0.7948 & 0.6256 & 0.3797 & 0.7620 & 0.5757 & 0.2877 \\
\hline & 4 & 16 & 0.5626 & 0.3675 & 0.1502 & 0.5275 & 0.3249 & 0.1126 & 0.4926 & 0.2753 & 0.0361 \\
\hline & 8 & 8 & 0.4554 & 0.2147 & 0.0023 & 0.4046 & 0.1809 & 0.0021 & 0.3695 & 0.1063 & 0.0017 \\
\hline
\end{tabular}




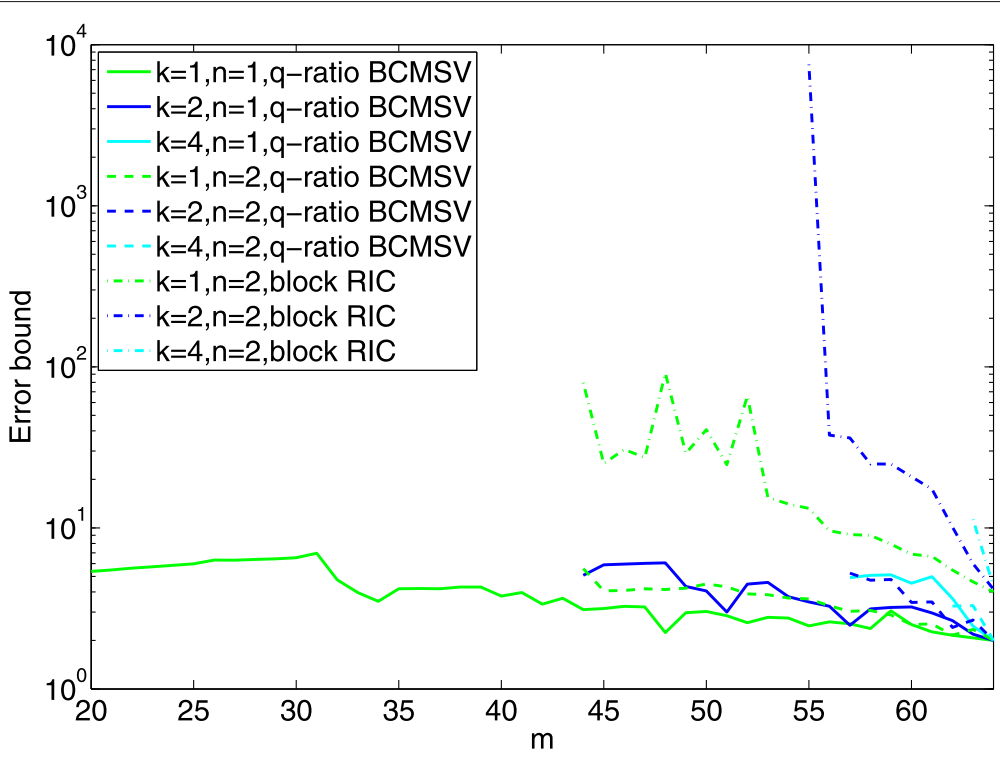

Fig. 2 The $q$-ratio BCMSV-based bounds and the block RIC-based bounds for Hadamard sub-matrices with $N=64, k=1,2,4, n=1,2$, and $q=1.8$

There are still some issues left for future work. For example, analogue to the case for the $q$-ratio CMSV, the geometrical property of the $q$-ratio BCMSV can be investigated to derive sufficient conditions and error bounds for block sparse signal recovery.

\section{Appendix - Proofs}

Basically, the main processes of proofs follow from those in [11] with extensions to block sparse signals. We list all the details here for the sake of completeness.

\section{A. 1}

Proof Suppose there exists $\mathbf{z} \in \operatorname{ker} A \backslash\{\mathbf{0}\}$ and $|S| \leq k$ such that $\left\|\mathbf{z}_{S}\right\|_{2,1} \geq\left\|\mathbf{z}_{S^{c}}\right\|_{2,1}$, then we have

$$
\begin{aligned}
& \|\mathbf{z}\|_{2,1}=\left\|\mathbf{z}_{S}\right\|_{2,1}+\left\|\mathbf{z}_{S^{c}}\right\|_{2,1} \leq 2\left\|\mathbf{z}_{S}\right\|_{2,1} \\
& \leq 2 k^{1-1 / q}\left\|\mathbf{z}_{S}\right\|_{2, q} \leq 2 k^{1-1 / q}\|\mathbf{z}\|_{2, q}, \quad \forall q \in(1, \infty],
\end{aligned}
$$

which is identical to $k \geq 2^{\frac{q}{1-q}} k_{q}(\mathbf{z}), \quad \forall q \in(1, \infty]$.

In contrast, suppose $\exists q \in(1, \infty]$ such that $k<$ $\min _{z \in \operatorname{ker} A \backslash\{\mathbf{0}\}} 2^{\frac{q}{1-q}} k_{q}(\mathbf{z})$, then $\left\|\mathbf{z}_{S}\right\|_{2,1}<\left\|\mathbf{z}_{S^{c}}\right\|_{2,1}$ holds for all $\mathbf{z} \in \operatorname{ker} A \backslash\{\mathbf{0}\}$ and $|S| \leq k$, which implies that the block null space property of order $k$ is fulfilled; thus, any block $k$-sparse signal $\mathbf{x}$ can be obtained via (7).

\section{A.2}

Proof (i) Prove the left hand side of (10):

For any $\mathbf{z} \in \mathbb{R}^{N} \backslash\{\mathbf{0}\}$ and $1<q_{2} \leq q_{1} \leq \infty$, suppose $k_{q_{1}}(\mathbf{z}) \leq s$, then we can get $\left(\frac{\|\mathbf{z}\|_{2,1}}{\|\mathbf{z}\|_{2, q_{1}}}\right)^{\frac{q_{1}}{q_{1}-1}} \leq s \Rightarrow$ $\|\mathbf{z}\|_{2,1} \leq s^{\frac{q_{1}-1}{q_{1}}}\|\mathbf{z}\|_{2, q_{1}} \leq s^{\frac{q_{1}-1}{q_{1}}}\|\mathbf{z}\|_{2, q_{2}}$. Since $\tilde{q}=\frac{q_{2}\left(q_{1}-1\right)}{q_{1}\left(q_{2}-1\right)}$ and $\frac{\|\mathbf{z}\|_{2,1}}{\|\mathbf{z}\|_{2, q_{2}}} \leq s^{\frac{q_{1}-1}{q_{1}}}$, we have

$$
k_{q_{2}}(\mathbf{z})=\left(\frac{\|\mathbf{z}\|_{2,1}}{\|\mathbf{z}\|_{2, q_{2}}}\right)^{\frac{q_{2}}{q_{2}-1}} \leq s^{\frac{q_{2}\left(q_{1}-1\right)}{q_{1}\left(q_{2}-1\right)}}=s^{\tilde{q}},
$$

from which we can infer

$$
\left\{\mathbf{z}: k_{q_{1}}(\mathbf{z}) \leq s\right\} \subseteq\left\{\mathbf{z}: k_{q_{2}}(\mathbf{z}) \leq s^{\tilde{q}}\right\} .
$$

Therefore, we can get the left hand side of (10) through

$$
\begin{aligned}
\beta_{q_{1}, s}(A) & =\min _{\mathbf{z} \neq \mathbf{0}, k_{q_{1}}(\mathbf{z}) \leq s} \frac{\|A \mathbf{z}\|_{2}}{\|\mathbf{z}\|_{2, q_{1}}} \geq \min _{\mathbf{z} \neq \mathbf{0}, k_{q_{2}}(\mathbf{z}) \leq s \tilde{q}} \frac{\|A \mathbf{z}\|_{2}}{\|\mathbf{z}\|_{2, q_{1}}} \\
& =\min _{\mathbf{z} \neq \mathbf{0}, k_{q_{2}}(\mathbf{z}) \leq s} \frac{\|A \mathbf{z}\|_{2}}{\|\mathbf{z}\|_{2, q_{2}}} \cdot \frac{\|\mathbf{z}\|_{2, q_{2}}}{\|\mathbf{z}\|_{2, q_{1}}} \\
& \geq \min _{\mathbf{z} \neq \mathbf{0}, k_{q_{2}}(\mathbf{z}) \leq s \tilde{q}} \frac{\|A \mathbf{z}\|_{2}}{\|\mathbf{z}\|_{2, q_{2}}}=\beta_{q_{2}, s^{\tilde{q}}}(A) .
\end{aligned}
$$

(ii) Verify the right hand side of (10):

Suppose $k_{q_{2}}(\mathbf{z}) \leq s^{\tilde{q}}$, for any $\mathbf{z} \in \mathbb{R}^{N} \backslash\{\mathbf{0}\}$, by using the non-increasing property of the $q$-ratio block sparsity with respect to $q$ and $q_{2} \leq q_{1} \leq \infty$, we have the following two inequalities: $\frac{\|\mathbf{z}\|_{2,1}}{\|\mathbf{z}\|_{2, \infty}}=k_{\infty}(\mathbf{z}) \leq k_{q_{2}}(\mathbf{z}) \leq s^{\tilde{q}}$ and $k_{q_{1}}(\mathbf{z}) \leq$ $k_{q_{2}}(\mathbf{z}) \leq s^{\tilde{q}}$. Since $1<q_{2} \leq q_{1} \leq \infty$, the former inequality implies that $\frac{\|\mathbf{z}\|_{2, q_{2}}}{\|\mathbf{z}\|_{2, q_{1}}} \leq \frac{\|\mathbf{z}\|_{2,1}}{\|\mathbf{z}\|_{2, \infty}} \leq s^{\tilde{q}} \Rightarrow \frac{\|\mathbf{z}\|_{2, q_{1}}}{\|\mathbf{z}\|_{2, q_{2}}} \geq s^{-\tilde{q}}$. The latter inequality implies that

$$
\left\{\mathbf{z}: k_{q_{2}}(\mathbf{z}) \leq s^{\tilde{q}}\right\} \subseteq\left\{\mathbf{z}: k_{q_{1}}(\mathbf{z}) \leq s^{\tilde{q}}\right\} .
$$


Therefore, we can obtain the right hand side of (10) through

$$
\begin{aligned}
\beta_{q_{2}, s^{\tilde{q}}}(A) & =\min _{\mathbf{z} \neq \mathbf{0}, k_{q_{2}}(\mathbf{z}) \leq s^{\tilde{q}}} \frac{\|A \mathbf{z}\|_{2}}{\|\mathbf{z}\|_{2, q_{2}}} \\
& \geq \min _{\mathbf{z} \neq \mathbf{0}, k_{q_{1}}(\mathbf{z}) \leq s^{\tilde{q}}} \frac{\|A \mathbf{z}\|_{2}}{\|\mathbf{z}\|_{2, q_{2}}} \\
& =\min _{\mathbf{z} \neq \mathbf{0}, k_{q_{1}}(\mathbf{z}) \leq s^{\tilde{q}}} \frac{\|A \mathbf{z}\|_{2}}{\|\mathbf{z}\|_{2, q_{1}}} \cdot \frac{\|\mathbf{z}\|_{2, q_{1}}}{\|\mathbf{z}\|_{2, q_{2}}} \\
& \geq \beta_{q_{1}, s^{\tilde{q}}}(A) \cdot s^{-\tilde{q}} .
\end{aligned}
$$

\section{A.3}

Proof The proof procedure follows from the similar arguments in $[6,10]$, and the procedure can be divided into two main steps.

Step 1: We first derive upper bounds of the $q$-ratio block sparsity of residual $\mathbf{h}=\hat{\mathbf{x}}-\mathbf{x}$ for all algorithms. As $\mathbf{x}$ is block $k$-sparse, we assume that $\operatorname{bsupp}(\mathbf{x})=S$ and $|S| \leq k$.

For the BBP and the BDS, since $\|\hat{\mathbf{x}}\|_{2,1}=\|\mathbf{x}+\mathbf{h}\|_{2,1}$ is the minimum among all $\mathbf{z}$ satisfying the constraints of $\mathrm{BBP}$ and BDS (including the true signal $\mathbf{x}$ ), we have

$$
\begin{aligned}
\|\mathbf{x}\|_{2,1} & \geq\|\hat{\mathbf{x}}\|_{2,1}=\|\mathbf{x}+\mathbf{h}\|_{2,1}=\left\|\mathbf{x}_{S}+\mathbf{h}_{S}\right\|_{2,1}+\left\|\mathbf{x}_{S^{c}}+\mathbf{h}_{S^{c}}\right\|_{2,1} \\
& \geq\left\|\mathbf{x}_{S}\right\|_{2,1}-\left\|\mathbf{h}_{S}\right\|_{2,1}+\left\|\mathbf{h}_{S^{c}}\right\|_{2,1} \\
& =\|\mathbf{x}\|_{2,1}-\left\|\mathbf{h}_{S}\right\|_{2,1}+\left\|\mathbf{h}_{S^{c}}\right\|_{2,1},
\end{aligned}
$$

which can be simplified to $\left\|\mathbf{h}_{S^{c}}\right\|_{2,1} \leq\left\|\mathbf{h}_{S}\right\|_{2,1}$. Thereby, we can obtain the following inequality:

$$
\begin{aligned}
& \|\mathbf{h}\|_{2,1}=\left\|\mathbf{h}_{S}\right\|_{2,1}+\left\|\mathbf{h}_{S^{c}}\right\|_{2,1} \leq 2\left\|\mathbf{h}_{S}\right\|_{2,1} \\
& \leq 2 k^{1-1 / q}\left\|\mathbf{h}_{S}\right\|_{2, q} \leq 2 k^{1-1 / q}\|\mathbf{h}\|_{2, q}, \quad \forall q \in(1, \infty],
\end{aligned}
$$

which is equivalent to

$$
k_{q}(\mathbf{h})=\left(\frac{\|\mathbf{h}\|_{2,1}}{\|\mathbf{h}\|_{2, q}}\right)^{\frac{q}{q-1}} \leq 2^{\frac{q}{q-1}} k
$$

For the group lasso, since the noise $\boldsymbol{\epsilon}$ satisfies $\left\|A^{T} \boldsymbol{\epsilon}\right\|_{2, \infty} \leq \kappa \mu$ for $\kappa \in(0,1)$ and $\hat{\mathbf{x}}$ is a solution of the group lasso, we have

$$
\frac{1}{2}\|A \hat{\mathbf{x}}-\mathbf{y}\|_{2}^{2}+\mu\|\hat{\mathbf{x}}\|_{2,1} \leq \frac{1}{2}\|A \mathbf{x}-\mathbf{y}\|_{2}^{2}+\mu\|\mathbf{x}\|_{2,1} .
$$

Substituting $\mathbf{y}$ by $A \mathbf{x}+\boldsymbol{\epsilon}$ leads to

$$
\begin{aligned}
\mu\|\hat{\mathbf{x}}\|_{2,1} & \leq \frac{1}{2}\|\boldsymbol{\epsilon}\|_{2}^{2}-\frac{1}{2}\|A(\hat{\mathbf{x}}-\mathbf{x})-\boldsymbol{\epsilon}\|_{2}^{2}+\mu\|\mathbf{x}\|_{2,1} \\
& =\frac{1}{2}\|\boldsymbol{\epsilon}\|_{2}^{2}-\frac{1}{2}\|A(\hat{\mathbf{x}}-\mathbf{x})\|_{2}^{2}+\langle A(\hat{\mathbf{x}}-\mathbf{x}), \boldsymbol{\epsilon}\rangle \\
& -\frac{1}{2}\|\boldsymbol{\epsilon}\|_{2}^{2}+\mu\|\mathbf{x}\|_{2,1} \\
& \leq\langle A(\hat{\mathbf{x}}-\mathbf{x}), \boldsymbol{\epsilon}\rangle+\mu\|\mathbf{x}\|_{2,1} \\
& =\left\langle\hat{\mathbf{x}}-\mathbf{x}, A^{T} \boldsymbol{\epsilon}\right\rangle+\mu\left\|_{\mathbf{x}}\right\|_{2,1} \\
& \leq\|\hat{\mathbf{x}}-\mathbf{x}\|_{2,1}\left\|A^{T} \boldsymbol{\epsilon}\right\|_{2, \infty}+\mu\left\|_{\mathbf{x}}\right\|_{2,1} \\
& \leq \kappa \mu\|\mathbf{h}\|_{2,1}+\mu\|\mathbf{x}\|_{2,1} .
\end{aligned}
$$

The last second inequality follows by applying CauchySchwarz inequality block wise and the last inequality can be written as

$$
\|\hat{\mathbf{x}}\|_{2,1} \leq \kappa\|\mathbf{h}\|_{2,1}+\|\mathbf{x}\|_{2,1} .
$$

Therefore, it holds that

$$
\begin{aligned}
\|\mathbf{x}\|_{2,1} & \geq\|\hat{\mathbf{x}}\|_{2,1}-\kappa\|\mathbf{h}\|_{2,1} \\
& =\left\|\mathbf{x}+\mathbf{h}_{S^{c}}+\mathbf{h}_{S}\right\|_{2,1}-\kappa\left\|\mathbf{h}_{S^{c}}+\mathbf{h}_{S}\right\|_{2,1} \\
& \geq\left\|\mathbf{x}+\mathbf{h}_{S^{c}}\right\|_{2,1}-\left\|\mathbf{h}_{S}\right\|_{2,1}-\kappa\left(\left\|\mathbf{h}_{S^{c}}\right\|_{2,1}+\left\|\mathbf{h}_{S}\right\|_{2,1}\right) \\
& =\|\mathbf{x}\|_{2,1}+(1-\kappa)\left\|\mathbf{h}_{S^{c}}\right\|_{2,1}-(1+\kappa)\left\|\mathbf{h}_{S}\right\|_{2,1},
\end{aligned}
$$

which can be simplified to

$$
\left\|\mathbf{h}_{S^{c}}\right\|_{2,1} \leq \frac{1+\kappa}{1-\kappa}\left\|\mathbf{h}_{S}\right\|_{2,1} .
$$

Thus, we can obtain

$$
\begin{aligned}
\|\mathbf{h}\|_{2,1} & =\left\|\mathbf{h}_{S^{c}}\right\|_{2,1}+\left\|\mathbf{h}_{S}\right\|_{2,1} \\
& \leq \frac{2}{1-\kappa}\left\|\mathbf{h}_{S}\right\|_{2,1} \\
& \leq \frac{2}{1-\kappa} k^{1-1 / q}\left\|\mathbf{h}_{S}\right\|_{2, q} \\
& \leq \frac{2}{1-\kappa} k^{1-1 / q}\|\mathbf{h}\|_{2, q},
\end{aligned}
$$

which can be reformulated by

$$
k_{q}(\mathbf{h})=\left(\frac{\|\mathbf{h}\|_{2,1}}{\|\mathbf{h}\|_{2, q}}\right)^{\frac{q}{q-1}} \leq\left(\frac{2}{1-\kappa}\right)^{\frac{q}{q-1}} k .
$$

Step 2: Obtain upper bound of $\|A \mathbf{h}\|_{2}$ and then construct the mixed $\ell_{2} / \ell_{q}$ norm and the mixed $\ell_{2} / \ell_{1}$ norm of the recovery error vector $\mathbf{h}$ via the $q$-ratio BCMSV for each algorithm.

(i) For the BBP, since both $\mathbf{x}$ and $\hat{\mathbf{x}}$ satisfy the constraint $\|\mathbf{y}-A \mathbf{z}\|_{2} \leq \zeta$, by using the triangle inequality, we can get

$$
\|A \mathbf{h}\|_{2}=\|A(\hat{\mathbf{x}}-\mathbf{x})\|_{2} \leq\|A \hat{\mathbf{x}}-\mathbf{y}\|_{2}+\|\mathbf{y}-A \mathbf{x}\|_{2} \leq 2 \zeta .
$$


Following from the definition of the $q$-ratio BCMSV and $k_{q}(\mathbf{h}) \leq 2^{\frac{q}{q-1}} k$, we have

$$
\beta_{q, 2^{\frac{q}{q-1}} k}(A)\|\mathbf{h}\|_{2, q} \leq\|A \mathbf{h}\|_{2} \leq 2 \zeta \Rightarrow\|\mathbf{h}\|_{2, q} \leq \frac{2 \zeta}{\beta_{q, 2^{\frac{q}{q-1}} k}(A)} .
$$

Furthermore, we can obtain $\|\mathbf{h}\|_{2,1} \leq \frac{4 k^{1-1 / q} \zeta}{\beta{ }_{q, 2} \frac{q}{q-1}{ }_{k}(A)}$ by using the property $\|\mathbf{h}\|_{2,1} \leq 2 k^{1-1 / q}\|\mathbf{h}\|_{2, q}$.

(ii) Similarly for the BDS, since both $\mathbf{x}$ and $\hat{\mathbf{x}}$ satisfy the constraint $\left\|A^{T}(\mathbf{y}-\mathbf{A z})\right\|_{2, \infty} \leq \mu$, we have

$\left\|A^{T} A \mathbf{h}\right\|_{2, \infty} \leq\left\|A^{T}(\mathbf{y}-A \hat{\mathbf{x}})\right\|_{2, \infty}+\left\|A^{T}(\mathbf{y}-A \mathbf{x})\right\|_{2, \infty} \leq 2 \mu$.

By applying the Cauchy-Schwarz inequality again as in Step 1, we obtain

$$
\begin{aligned}
& \|A \mathbf{h}\|_{2}^{2}=\langle A \mathbf{h}, A \mathbf{h}\rangle=\left\langle\mathbf{h}, A^{T} A \mathbf{h}\right\rangle \\
& \leq\|\mathbf{h}\|_{2,1}\left\|A^{T} A \mathbf{h}\right\|_{2, \infty} \leq 2 \mu\|\mathbf{h}\|_{2,1} .
\end{aligned}
$$

At last, with the definition of the $q$-ratio BCMSV, $k_{q}(\mathbf{h}) \leq$ $2^{\frac{q}{q-1}} k$ and $\|\mathbf{h}\|_{2,1} \leq 2 k^{1-1 / q}\|\mathbf{h}\|_{2, q}$, we get the upper bounds of the mixed $\ell_{2} / \ell_{q}$ norm and the mixed $\ell_{2} / \ell_{1}$ norm for $\mathbf{h}$ :

$$
\begin{aligned}
& \beta_{q, 2^{\frac{q}{q-1} k}}^{2}(A)\|\mathbf{h}\|_{2, q}^{2} \leq\|A \mathbf{h}\|_{2}^{2} \leq 2 \mu\|\mathbf{h}\|_{2,1} \leq 4 \mu k^{1-1 / q}\|\mathbf{h}\|_{2, q} \\
& \Rightarrow\|\mathbf{h}\|_{2, q} \leq \frac{4 k^{1-1 / q}}{\beta_{q, 2^{\frac{q}{q-1} k}}^{2}(A)} \mu
\end{aligned}
$$

and $\|\mathbf{h}\|_{2,1} \leq 2 k^{1-1 / q}\|\mathbf{h}\|_{2, q} \leq \frac{8 k^{2-2 / q}}{\beta^{2}{\frac{q}{q, 2^{q-1}} k}^{(A)}} \mu$.

(iii) For the group lasso, with $\left\|A^{T} \boldsymbol{\epsilon}\right\|_{2, \infty} \leq \kappa \mu$, we have

$$
\begin{aligned}
\left\|A^{T} A \mathbf{h}\right\|_{2, \infty} & \leq\left\|A^{T}(\mathbf{y}-A \mathbf{x})\right\|_{2, \infty}+\left\|A^{T}(\mathbf{y}-A \hat{\mathbf{x}})\right\|_{2, \infty} \\
& \leq\left\|A^{T} \boldsymbol{\epsilon}\right\|_{2, \infty}+\left\|A^{T}(\mathbf{y}-A \hat{\mathbf{x}})\right\|_{2, \infty} \\
& \leq \kappa \mu+\left\|A^{T}(\mathbf{y}-A \hat{\mathbf{x}})\right\|_{2, \infty} .
\end{aligned}
$$

Moreover, since $\hat{\mathbf{x}}$ is the solution of the group lasso, the optimality condition yields that

$$
A^{T}(\mathbf{y}-A \hat{\mathbf{x}}) \in \mu \partial\|\hat{\mathbf{x}}\|_{2,1},
$$

where the sub-gradients in $\partial\|\hat{\mathbf{x}}\|_{2,1}$ for the $i$ th block are $\hat{\mathbf{x}}_{i} /\left\|\hat{\mathbf{x}}_{i}\right\|_{2}$ if $\hat{\mathbf{x}}_{i} \neq 0$ and is some vector $\mathbf{g}$ satisfying $\|\mathbf{g}\|_{2} \leq$ 1 if $\hat{\mathbf{x}}_{i}=0$ (which follows from the definition of subgradient). Thus, we have $\left\|A^{T}(\mathbf{y}-A \hat{\mathbf{x}})\right\|_{2, \infty} \leq \mu$, which leads to

$$
\left\|A^{T} A \mathbf{h}\right\|_{2, \infty} \leq(\kappa+1) \mu .
$$

Following the inequality (35), we get

$$
\|A \mathbf{h}\|_{2}^{2} \leq(\kappa+1) \mu\|\mathbf{h}\|_{2,1} \text {. }
$$

As a result, since $k_{q}(\mathbf{h}) \leq\left(\frac{2}{1-\kappa}\right)^{\frac{q}{q-1}} k$ and $\|\mathbf{h}\|_{2,1} \leq$ $\frac{2}{1-\kappa} k^{1-1 / q}\|\mathbf{h}\|_{2, q}$, we can obtain

$$
\begin{aligned}
& \beta_{q,\left(\frac{2}{1-\kappa}\right)^{2} \frac{q}{q-1} k}(A)\|\mathbf{h}\|_{2, q}^{2} \leq\|A \mathbf{h}\|_{2}^{2} \leq(\kappa+1) \mu\|\mathbf{h}\|_{2,1} \\
& \leq \mu \frac{2(\kappa+1)}{1-\kappa} k^{1-1 / q}\|\mathbf{h}\|_{2, q},
\end{aligned}
$$

which is equivalent to

$$
\|\mathbf{h}\|_{2, q} \leq \frac{k^{1-1 / q}}{\beta^{2},\left(\frac{2}{1-\kappa}\right)^{\frac{q}{q-1} k}(A)} \cdot \frac{2(\kappa+1)}{1-\kappa} \mu
$$

and $\|\mathbf{h}\|_{2,1} \leq \frac{1+\kappa}{(1-\kappa)^{2}} \cdot \frac{4 k^{2-2 / q}}{\beta^{2}{ }_{q,\left(\frac{2}{1-\kappa}\right)^{\frac{q}{q-1}} k}{ }^{(A)}} \mu$.

A.4

Proof Since the infimum of $\phi_{k}(\mathbf{x})$ is achieved by an block $k$-sparse signal $\mathbf{z}$ whose non-zero blocks equal to the largest $k$ blocks, indexed by $S$, of $\mathbf{x}$, so $\phi_{k}(\mathbf{x})=\left\|\mathbf{x}_{S^{c}}\right\|_{2,1}$ and let $\mathbf{h}=\hat{\mathbf{x}}-\mathbf{x}$. Similar as the proof procedure for Theorem 1 , the derivations also have two steps.

Step 1: For all algorithms, bound $\|\mathbf{h}\|_{2,1}$ via $\|\mathbf{h}\|_{2, q}$ and $\phi_{k}(\mathbf{x})$.

First for the BBP and the BDS, since $\|\hat{\mathbf{x}}\|_{2,1}=\|\mathbf{x}+\mathbf{h}\|_{2,1}$ is the minimum among all $\mathbf{z}$ satisfying the constraints of the BBP and the BDS, we have

$$
\begin{aligned}
\left\|\mathbf{x}_{S}\right\|_{2,1}+\left\|\mathbf{x}_{S^{c}}\right\|_{2,1} & =\|\mathbf{x}\|_{2,1} \geq\|\hat{\mathbf{x}}\|_{2,1}=\|\mathbf{x}+\mathbf{h}\|_{2,1} \\
& =\left\|\mathbf{x}_{S}+\mathbf{h}_{S}\right\|_{2,1}+\left\|\mathbf{x}_{S^{c}}+\mathbf{h}_{S^{c}}\right\|_{2,1} \\
& \geq\left\|\mathbf{x}_{S}\right\|_{2,1}-\left\|\mathbf{h}_{S}\right\|_{2,1}+\left\|\mathbf{h}_{S^{c}}\right\|-\left\|\mathbf{x}_{S^{c}}\right\|_{2,1},
\end{aligned}
$$

which is equivalent to

$$
\left\|\mathbf{h}_{S^{c}}\right\|_{2,1} \leq\left\|\mathbf{h}_{S}\right\|_{2,1}+2\left\|\mathbf{x}_{S^{c}}\right\|_{2,1}=\left\|\mathbf{h}_{S}\right\|_{2,1}+2 \phi_{k}(\mathbf{x}) .
$$

In consequence, we can get

$$
\begin{aligned}
\|\mathbf{h}\|_{2,1} & =\left\|\mathbf{h}_{S}\right\|_{2,1}+\left\|\mathbf{h}_{S^{c}}\right\|_{2,1} \\
& \leq 2\left\|\mathbf{h}_{S}\right\|_{2,1}+2 \phi_{k}(\mathbf{x}) \\
& \leq 2 k^{1-1 / q}\left\|\mathbf{h}_{S}\right\|_{2, q}+2 \phi_{k}(\mathbf{x}) \\
& \leq 2 k^{1-1 / q}\|\mathbf{h}\|_{2, q}+2 \phi_{k}(\mathbf{x}) .
\end{aligned}
$$

As for the group lasso, by using (33), we can obtain

$$
\begin{aligned}
\left\|\mathbf{x}_{S}\right\|_{2,1}+\left\|\mathbf{x}_{S^{c}}\right\|_{2,1} & =\|\mathbf{x}\|_{2,1} \geq\|\hat{\mathbf{x}}\|_{2,1}-\kappa\|\mathbf{h}\|_{2,1} \\
& \geq\left\|\mathbf{x}_{S}+\mathbf{x}_{S^{c}}+\mathbf{h}_{S}+\mathbf{h}_{S^{c}}\right\|_{2,1} \\
& -\kappa\left\|\mathbf{h}_{S}+\mathbf{h}_{S^{c}}\right\|_{2,1} \\
& \geq\left\|\mathbf{x}_{S}+\mathbf{h}_{S^{c}}\right\|_{2,1}-\left\|\mathbf{x}_{S^{c}}\right\|_{2,1} \\
& -\left\|\mathbf{h}_{S}\right\|_{2,1}-\kappa\left\|\mathbf{h}_{S}\right\|_{2,1}-\kappa\left\|_{S^{c}}\right\|_{2,1} \\
& =\left\|\mathbf{x}_{S}\right\|_{2,1}+(1-\kappa)\left\|\mathbf{h}_{S^{c}}\right\|_{2,1} \\
& -\left\|\mathbf{x}_{S^{c}}\right\|_{2,1}-(1+\kappa)\left\|\mathbf{h}_{S}\right\|_{2,1},
\end{aligned}
$$


which points to that

$$
\left\|\mathbf{h}_{S^{c}}\right\|_{2,1} \leq \frac{1+\kappa}{1-\kappa}\left\|\mathbf{h}_{S}\right\|_{2,1}+\frac{2}{1-\kappa}\left\|\mathbf{x}_{S^{c}}\right\|_{2,1} .
$$

Therefore, we have

$$
\begin{aligned}
\|\mathbf{h}\|_{2,1} & \leq\left\|\mathbf{h}_{S}\right\|_{2,1}+\left\|\mathbf{h}_{S^{c}}\right\|_{2,1} \\
& \leq \frac{2}{1-\kappa}\left\|\mathbf{h}_{S}\right\|_{2,1}+\frac{2}{1-\kappa}\left\|\mathbf{x}_{S^{c}}\right\|_{2,1} \\
& \leq \frac{2}{1-\kappa} k^{1-1 / q}\|\mathbf{h}\|_{2, q}+\frac{2}{1-\kappa} \phi_{k}(\mathbf{x}) .
\end{aligned}
$$

Step 2: Verify that the $q$-ratio block sparsity of $\mathbf{h}$ has lower bound in the form of $\|\mathbf{h}\|_{2, q}$ for each algorithm, when $\|\mathbf{h}\|_{2, q}$ is larger than the part of recovery bounds caused by the measurement error.

(i) For the BBP, we assume that $\mathbf{h} \neq \mathbf{0}$ and $\|\mathbf{h}\|_{2, q}>$ $\frac{2 \zeta}{\beta \frac{q}{q, 4}(A)}$; otherwise, (17) holds trivially. Since $\|A \mathbf{h}\|_{2} \leq$ $2 \zeta$ (see (34)), we have $\|\mathbf{h}\|_{2, q}>\frac{\|A \mathbf{h}\|_{2}}{\beta \frac{q}{q, 4}(A)}$. Then, it holds that

$$
\frac{\|A \mathbf{h}\|_{2}}{\|\mathbf{h}\|_{2, q}}<\beta_{q, 4^{\frac{q}{q-1}} k}(A)=\min _{\mathbf{h} \neq \mathbf{0}, k_{q}(\mathbf{h}) \leq 4^{\frac{q}{q-1} k}} \frac{\|A \mathbf{h}\|_{2}}{\|\mathbf{h}\|_{2, q}},
$$

which implies that

$$
k_{q}(\mathbf{h})>4^{\frac{q}{q-1}} k \Rightarrow\|\mathbf{h}\|_{2,1}>4 k^{1-1 / q}\|\mathbf{h}\|_{2, q} .
$$

Combining (40), we have $\|\mathbf{h}\|_{2, q}<k^{1 / q-1} \phi_{k}(\mathbf{x})$, which completes the proof for (17). The error bound of the mixed $\ell_{2} / \ell_{1}$ norm (18) follows immediately from (17) and (40).

(ii) As for the BDS, similarly we assume $\mathbf{h} \neq \mathbf{0}$ and $\|\mathbf{h}\|_{2, q}>\frac{8 k^{1-1 / q}}{\beta^{2} \frac{q}{q, 4}(A)} \mu$; otherwise, (19) holds trivially. As $\|A \mathbf{h}\|_{2}^{2} \leq 2 \mu\|\mathbf{h}\|_{2,1}$ (see (35)), we have $\|\mathbf{h}\|_{2, q}>$ $\frac{4 k^{1-1 / q}}{\beta^{2}{ }_{q, 4}^{\frac{q}{q-1}}(A)} \cdot \frac{\|A \mathbf{h}\|_{2}^{2}}{\|\mathbf{h}\|_{2,1}}$. Then, we can get

$$
\beta_{q, 4^{2} \frac{q}{q-1} k}(A)=\min _{\mathbf{h} \neq \mathbf{0}, k_{q}(\mathbf{h}) \leq 4} \frac{\|A \mathbf{h}\|_{2}^{2}}{\|\mathbf{q}\|_{2, q}^{2}}>\frac{\|A \mathbf{h}\|_{2}^{2}}{\|\mathbf{h}\|_{2, q}^{2}}\left(\frac{4^{\frac{q}{q-1}} k}{k_{q}(\mathbf{h})}\right)^{1-1 / q},
$$

which implies that

$$
k_{q}(\mathbf{h})>4^{\frac{q}{q-1}} k \Rightarrow\|\mathbf{h}\|_{2,1}>4 k^{1-1 / q}\|\mathbf{h}\|_{2, q} .
$$

Combining (40), we have $\|\mathbf{h}\|_{2, q}<k^{1 / q-1} \phi_{k}(\mathbf{x})$, which completes the proof for (19). (20) holds as a result of (19) and (40).

(iii) For the group lasso, we assume that $\mathbf{h} \neq \mathbf{0}$ and $\|\mathbf{h}\|_{2, q}>\frac{1+\kappa}{1-\kappa} \cdot \frac{4 k^{1-1 / q}}{\beta^{2}{ }_{q,\left(\frac{4}{1-\kappa}\right)^{\frac{q}{q-1}} k}(A)} \mu$; otherwise, (21) holds trivially. Since in this case $\|A \mathbf{h}\|_{2}^{2} \leq(1+\kappa) \mu\|\mathbf{h}\|_{2,1}$ (see (36)), we have $\|\mathbf{h}\|_{2, q}>\frac{4 k^{1-1 / q}}{(1-\kappa) \beta^{2}} \frac{\|A \mathbf{h}\|_{2}^{2}}{q,\left(\frac{4}{1-\kappa}\right)^{\frac{q}{q-1} k}}$, which leads

to

$$
\begin{aligned}
\beta_{q,\left(\frac{4}{1-\kappa}\right)^{\frac{q}{q-1}} k}^{2}(A) & \min _{\mathbf{h} \neq \mathbf{0}, k_{q}(\mathbf{h}) \leq\left(\frac{4}{1-\kappa}\right)^{\frac{q}{q-1}} k} \frac{\|A \mathbf{h}\|_{2}^{2}}{\|\mathbf{h}\|_{2, q}^{2}} \\
& >\frac{\|A \mathbf{h}\|_{2}^{2}}{\|\mathbf{h}\|_{2, q}^{2}}\left(\frac{\left(\frac{4}{1-\kappa}\right)^{\frac{q}{q-1}} k}{k_{q}(\mathbf{h})}\right)^{1-\frac{1}{q}} \\
& \Rightarrow k_{q}(\mathbf{h})>\left(\frac{4}{1-\kappa}\right)^{\frac{q}{q-1}} k \\
& \Rightarrow\|\mathbf{h}\|_{2,1}>\frac{4}{1-\kappa} k^{1-1 / q}\|\mathbf{h}\|_{2, q} .
\end{aligned}
$$

Combining (42), we have $\|\mathbf{h}\|_{2, q}<k^{1 / q-1} \phi_{k}(\mathbf{x})$, which completes the proof for (21). Consequently, (22) is obtained via (21) and (42).

\section{Abbreviations}

BBP: Block BP; BCMSV: q-ratio block constrained minimal singular values; BDS: Block DS; BP: Basis pursuit; CMSV: $\ell 1$-constrained minimal singular value; CS: Compressive sensing; DS: Dantzig selector; NSP: Null space property; RIC: Restricted isometry constant; RIP: Restricted isometry property

\section{Acknowledgements}

This work is supported by the Swedish Research Council grant (Reg.No. 340-2013-5342).

\section{Authors' contributions}

The authors read and approved the final manuscript.

Availability of data and materials

Please contact the author for data request.

\section{Competing interests}

The authors declare that they have no competing interests.

\section{Author details}

${ }^{1}$ Department of Mathematics and Mathematical Statistics, Umeå University, Umeå, Sweden. ${ }^{2}$ Department of Statistics, Zhejiang University City College, Hangzhou, China.

Received: 9 July 2019 Accepted: 28 October 2019 Published online: 27 November 2019

\section{References}

1. D. L. Donoho, Compressed sensing. IEEE Trans. Inf. Theory. 52(4), 1289-1306 (2006)

2. E. J. Candes, J. Romberg, T. Tao, Stable signal recovery from incomplete and inaccurate measurements. Commun. Pure Appl. Math. 59(8), 1207-1223 (2006)

3. S. Foucart, H. Rauhut, A Mathematical Introduction to Compressive Sensing. (Springer, 2013). https://doi.org/10.1007/978-0-8176-4948-7_1

4. A. S. Bandeira, E. Dobriban, D. G. Mixon, W. F. Sawin, Certifying the restricted isometry property is hard. IEEE Trans. Info. Theory. 59(6), 3448-3450 (2013)

5. A. M. Tillmann, M. E. Pfetsch, The computational complexity of the restricted isometry property, the nullspace property, and related concepts in compressed sensing. IEEE Trans. Inf. Theory. 60(2), 1248-1259 (2014)

6. G. Tang, A. Nehorai, Performance analysis of sparse recovery based on constrained minimal singular values. IEEE Trans. Sig. Process. 59(12) 5734-5745 (2011)

7. S. S. Chen, D. L. Donoho, M. A. Saunders, Atomic decomposition by basis pursuit. SIAM J. Sci. Comput. 20, 33-61 (1998) 
8. E. J. Candes, T. Tao, The Dantzig selector: statistical estimation when $p$ is much larger than $\boldsymbol{n}$. Ann. Stat., 2313-2351 (2007). https://doi.org/10.1214/ 009053606000001523

9. R. Tibshirani, Regression shrinkage and selection via the lasso. J. R. Stat Soc. Ser B (Methodol), 267-288 (1996). https://doi.org/10.1111/j.14679868.2011.00771.x

10. G. Tang, A. Nehorai, Computable performance bounds on sparse recovery. IEEE Trans. Sig. Process. 63(1), 132-141 (2015)

11. Z. Zhou, J. Yu, Sparse recovery based on q-ratio constrained minimal singular values. Sig. Process. 155, 247-258 (2019)

12. Z. Zhou, J. Yu, On q-ratio cmsv for sparse recovery. Sig. Process (2019). https://doi.org/10.1016/j.sigpro.2019.07.003

13. R. G. Baraniuk, V. Cevher, M. F. Duarte, C. Hegde, Model-based compressive sensing. IEEE Trans. Inf. Theory. 56(4), 1982-2001 (2010)

14. Y. C. Eldar, M. Mishali, Robust recovery of signals from a structured union of subspaces. IEEE Trans. Inf. Theory. 55(11), 5302-5316 (2009)

15. H. Zamani, H. Bahrami, P. Mohseni, in Proc. IEEE Biomedical Circuits and Systems Conf. (BioCAS). On the use of compressive sensing (Cs) exploiting block sparsity for neural spike recording, (2016), pp. 228-231. https://doi. org/10.1109/biocas.2016.7833773

16. Y. Gao, M. Ma, A new bound on the block restricted isometry constant in compressed sensing. J. Inequalities Appl. 2017(1), 174-174 (2017)

17. G. Tang, A. Nehorai, Semidefinite programming for computable performance bounds on block-sparsity recovery. IEEE Trans. Sig. Process. 64(17), 4455-4468 (2016)

18. Z. Zhou, J. Yu, Estimation of block sparsity in compressive sensing (2017). arXiv preprint arXiv:1701.01055

19. M. E. Lopes, in International Conference on Machine Learning. Estimating unknown sparsity in compressed sensing, (2013), pp. 217-225. http:// proceedings.mlr.press/v28/lopes13.pdf

20. M. E. Lopes, Unknown sparsity in compressed sensing: denoising and inference. IEEE Trans. Inf. Theory. 62(9), 5145-5166 (2016)

21. Y. Plan, R. Vershynin, One-bit compressed sensing by linear programming Commun. Pure Appl. Math. 66(8), 1275-1297 (2013)

22. R. Vershynin, in Sampling Theory, a Renaissance. Estimation in high dimensions: a geometric perspective (Springer, Cham, 2015), pp. 3-66

23. M. Stojnic, F. Parvaresh, B. Hassibi, On the reconstruction of block-sparse signals with an optimal number of measurements. IEEE Trans. Sig. Process. 57, 3075-3085 (2009)

24. H. Liu, J. Zhang, X. Jiang, J. Liu, in Proceedings of the Thirteenth International Conference on Artificial Intelligence and Statistics. Proceedings of Machine Learning Research, vol. 9, ed. by Y. W. Teh, M. Titterington. The group Dantzig selector (PMLR, Chia Laguna Resort, Sardinia, 2010), pp. 461-468. http://proceedings.mlr.press/v9/liu10a.html

25. R. Garg, R. Khandekar, in Proceedings of the Fourteenth International Conference on Artificial Intelligence and Statistics. Proceedings of Machine Learning Research, vol. 15, ed. by G. Gordon, D. Dunson, and M. Dudík. Block-sparse solutions using kernel block rip and its application to group lasso (PMLR, Fort Lauderdale, 2011), pp. 296-304. http://proceedings.mlr. press/v15/garg11a.html

26. T. Lipp, S. Boyd, Variations and extension of the convex-concave procedure. Optim. Eng. 17(2), 263-287 (2016)

27. N. Rao, B. Recht, R. Nowak, in Proceedings of the Fifteenth International Conference on Artificial Intelligence and Statistics. Proceedings of Machine Learning Research, vol. 22, ed. by N. D. Lawrence, M. Girolami. Universal measurement bounds for structured sparse signal recovery (PMLR, La Palma, 2012), pp. 942-950. http://proceedings.mlr.press/v22/rao12.html

\section{Publisher's Note}

Springer Nature remains neutral with regard to jurisdictional claims in published maps and institutional affiliations. 